\title{
Differential effects of defibrillation on systemic and cardiac sympathetic activity
}

\author{
F Bode, U Wiegand, W Raasch, G Richardt, J Potratz
}

\begin{abstract}
Objective-To assess the effect of defibrillation shocks on cardiac and circulating catecholamines.

Design-Prospective examination of myocardial catecholamine balance during dc shock by simultaneous determination of arterial and coronary sinus plasma concentrations. Internal countershocks (10$34 \mathrm{~J})$ were applied in 30 patients after initiation of ventricular fibrillation for a routine implantable cardioverter defibrillator test. Another 10 patients were externally cardioverted (50-360 J) for atrial fibrillation.
\end{abstract}

Main outcome measures-Transcardiac noradrenaline, adrenaline, and lactate gradients immediately after the shock.

Results-After internal shock, arterial noradrenaline increased from a mean (SD) of $263(128) \mathrm{pg} / \mathrm{ml}$ at baseline to 370 (148) $\mathrm{pg} / \mathrm{ml}(\mathrm{p}=\mathbf{0 . 0 0 1})$, while coronary sinus noradrenaline fell from 448 (292) to 363 (216) $\mathrm{pg} / \mathrm{ml}(\mathrm{p}=0.01)$, reflecting a shift from cardiac net release to net uptake. After external shock delivery, there was a similar increase in arterial noradrenaline, from 260 (112) to 459 (200) $\mathrm{pg} / \mathrm{ml} \quad(\mathrm{p}=0.03)$, while coronary sinus noradrenaline remained unchanged. Systemic adrenaline increased 11-fold after external shock $(p=0.01)$, outlasting the threefold rise following internal shock $(p=0.001)$. In both groups, a negative transmyocardial adrenaline gradient at baseline decreased further, indicating enhanced myocardial uptake. Cardiac lactate production occurred after ventricular fibrillation and internal shock, but not after external cardioversion, so the neurohumoral changes resulted from the defibrillation process and not from alterations in oxidative metabolism.

Conclusions-A dc shock induces marked systemic sympathoadrenal and sympathoneuronal activation, but attenuates cardiac sympathetic activity. This might promote the transient myocardial depression observed after electrical discharge to the heart.

(Heart 1998;79:560-567)

Keywords: defibrillation; autonomic cardiac function; catecholamines; lactate

Defibrillatory shocks alter cardiac function temporarily. Multiple forms of conduction disturbance and bradyarrhythmias, as well as tachyarrhythmias, can evolve following electri- cal cardioversion. ${ }^{1-3}$ Rhythm abnormalities have been attributed to the depolarising action of the shock on the myocardium, ${ }^{45}$ but they may also be due to neurohumoral influences. ${ }^{6-8}$ Sympathetic activity is an important modulator of the electrophysiological properties and contractile function of the heart. Enhancement of myocardial excitability, shortening of action potential duration and refractory period, ${ }^{9}$ and the occurrence of ectopic rhythms after shock have all been attributed to adrenergic stimulation. ${ }^{7}$ Therefore cardiac sympathetic tone may influence the stability of a regular rhythm achieved by shock treatment. When ineffective shocks fail to terminate an arrhythmia, it is possible that the release of endogenous catecholamines affects the outcome of further treatment by lowering the defibrillation threshold. ${ }^{10}{ }^{11}$

Cardiac sympathetic activity could be affected by electric stimulation of afferent and efferent nerves during shock delivery, it might be increased by pain or preceding hypotension ${ }^{12}$ through reflex mechanisms, or it could be attenuated by short term ischaemia. ${ }^{1314}$ At present there are no conclusive data on cardiac sympathetic responses to countershocks. Postshock increases in arterial and venous noradrenaline concentrations have been reported in humans, ${ }^{915}$ suggesting there is an increment in overall sympathetic tone. As sympathetic nervous responses are regionalised $^{16}$ and myocardial release of the transmitter accounts for only a small proportion of circulating noradrenaline,${ }^{17} \mathrm{car}-$ diac sympathetic activity cannot be inferred from systemic noradrenaline concentrations. Indeed, an animal study on postshock cardiac metabolism outlined an antiadrenergic mechanism attenuating myocardial noradrenaline release and thereby mediating postdefibrillation bradyarrhythmia and haemodynamic depression. ${ }^{18}$ However, measurement of cardiac catecholamines to prove this hypothesis has not yet been performed.

In this study we sought to determine whether shock treatment leads to cardiac adrenergic activation in humans. Postshock plasma catecholamine concentrations were analysed in coronary sinus effluent to obtain a more accurate estimate of catecholamine levels in myocardial tissue. The effect of dc shocks on transcardiac catecholamine balance was assessed in two frequent clinical applicationscardioversion from atrial fibrillation, and termination of ventricular fibrillation. This allowed us to differentiate the effect of isolated elective shocks from the impact of shocks required to terminate circulatory collapse. 
Table 1 Patient characteristics

\begin{tabular}{|c|c|c|}
\hline & $\begin{array}{l}\text { Ventricular } \\
\text { fibrillation }\end{array}$ & $\begin{array}{l}\text { Atrial } \\
\text { fibrillation }\end{array}$ \\
\hline Patients (n) & 30 & 10 \\
\hline Women (n) & 3 & 3 \\
\hline Mean (SD) age (years) & $58(7)$ & $57(14)$ \\
\hline Range & $41-76$ & $29-74$ \\
\hline \multicolumn{3}{|l|}{ Cardiovascular disease (n) } \\
\hline Coronary artery disease & 25 & 4 \\
\hline One vessel & 14 & 0 \\
\hline Two vessel & 4 & 2 \\
\hline Three vessel & 7 & 2 \\
\hline Previous myocardial infarction & 23 & 3 \\
\hline Anterior (-lateral) wall & 15 & 3 \\
\hline Posterior (-lateral) wall & 11 & 1 \\
\hline Dilated cardiomyopathy & 4 & 0 \\
\hline Aortocoronary venous bypass & 3 & 2 \\
\hline Left ventricular ejection fraction (\%) & $47 \quad(20)$ & $60(15)^{\star}$ \\
\hline NYHA class & $2.1(0.6)$ & $1.9(0.7)$ \\
\hline Digitalis medication (n) & 7 & 9 \\
\hline \multicolumn{3}{|l|}{ Analgesics/sedatives } \\
\hline Midazolam $(\mu \mathrm{g} / \mathrm{kg})$ & $116(43)$ & $71 \quad(26)$ \\
\hline Fentanyl $(\mu \mathrm{g} / \mathrm{kg})$ & $34(12)$ & $31(10)$ \\
\hline Etomidate $(\mu \mathrm{g} / \mathrm{kg})$ & 0 & $119(53)$ \\
\hline
\end{tabular}

Values are means (SD).

${ }^{\star} \mathrm{p}<0.05$ for intergroup difference.

NYHA, New York Heart Association.

\section{Methods}

PATIENTS

After approval by the institutional ethics review committee and informed consent was obtained, 40 patients participated in the study (six women, 34 men; mean (SD) age 57.5 (9.1) years).

Thirty subjects with implantable cardioverter defibrillators (ICD) were studied during a routine predischarge test of the device (PCD Medtronic, Minneapolis, Minnesota, USA). All had received a non-thoracotomy, single transvenous lead system (Medtronic Transvene system) with biphasic shock delivery in an "active can" configuration. The defibrillation coil was placed in the apical right ventricle. Indications were survival after ventricular fibrillation in 13 patients and sustained ventricular tachycardia with drug inefficacy or intolerance in 17 patients.

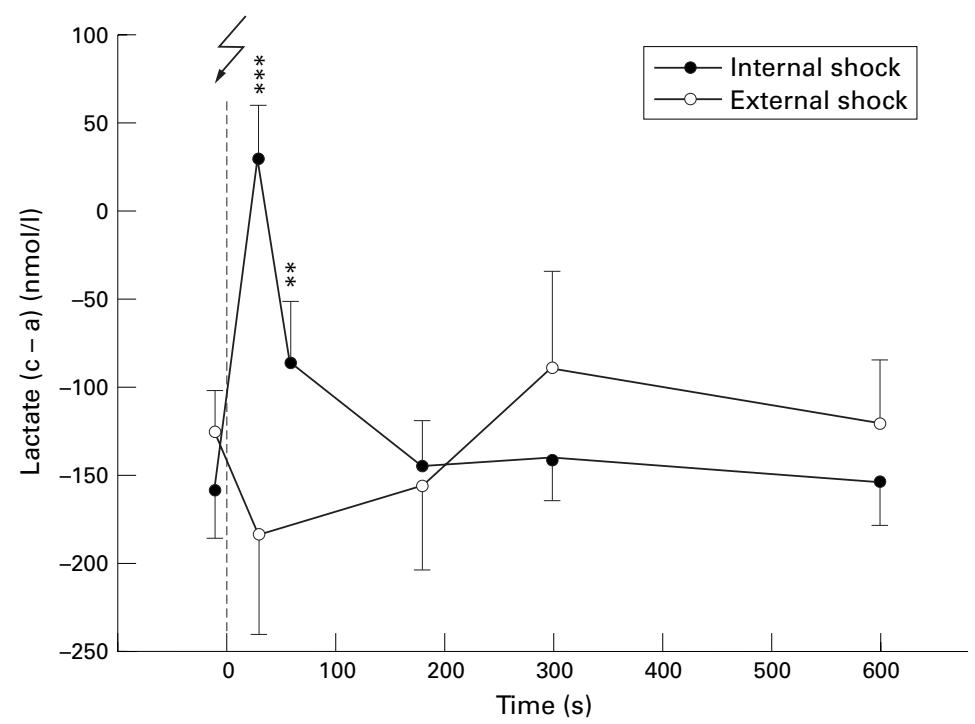

Figure 1 Coronary venous-arterial (c-a) lactate difference. Ventricular fibrillation terminated by an internal countershock shifted the negative transcardiac lactate gradient towards positive, signifying myocardial lactate production. In contrast, transcardiac lactate balance was not significantly affected by external shock delivery to terminate atrial fibrillation. Arrow indicates shock application. Values are means, error bars $=S E M$. ${ }_{\star \star} p<0.01 ;{ }^{\star \star *} p<0.001 v$ baseline.
A group of 10 patients underwent external cardioversion from atrial fibrillation. Synchronised dc shocks were generated by a Lifepak 10 (Physio-Control, Redmond, Oregon, USA) and delivered by left paravertebral and left parasternal patch electrodes.

In both groups, $\beta$ blocking agents and antiarrhythmic drugs (except digitalis glycosides) were withheld for two days before the study procedure. Myocardial infarction within the last three months was a reason for exclusion.

\section{CATHETERISATION PROCEDURE}

A 7 F NIH catheter (Cordis, Miami, Florida, USA) was introduced through a left or right brachial vein into the coronary sinus at least $4 \mathrm{~cm}$ beyond the orifice. Position stability was confirmed by repeated fluoroscopic control during the study. A $5 \mathrm{~F}$ introducer system (Cordis) was advanced into the right femoral artery to obtain blood samples and monitor systemic arterial pressure.

STUDY PROTOCOL

All patients were fasted overnight and premedicated with diazepam $10 \mathrm{mg}$. In the ICD group, sedation and analgesia was achieved by intravenous midazolam and fentanyl. Full blood oxygen saturation was ensured by face mask supply and continuous monitoring. Ventricular fibrillation was induced by either $50 \mathrm{~Hz}$ burst stimulation or $0.4 \mathrm{~J} \mathrm{~T}$ wave shock, delivered through the transvenous ICD system. Following detection of fibrillation by the device, an internal countershock was applied. To assure defibrillation efficiency, shock energy was programmed according to the individual defibrillation threshold found during implantation. Blood samples were collected simultaneously from the coronary sinus effluent and femoral artery directly before initiation of ventricular fibrillation, within 30 seconds after termination, and 1, 3, 5, and 10 minutes postshock.

In patients undergoing cardioversion from atrial fibrillation, instrumentation was performed as described above and etomidate was added to the anaesthetic regimen. Blood sampling was performed immediately before, within 30 seconds of, and 3, 5, and 10 minutes after successful atrial defibrillation in an identical fashion to that previously described. Cardioverting energy was varied among the patients.

In the sequence of blood collection, samples for lactate were obtained immediately after the samples for catecholamines. Approximately $100 \mathrm{ml}$ of blood were required for analysis.

CATECHOLAMINE AND LACTATE MEASUREMENTS For the assessment of neurohormones a minimum of $6 \mathrm{ml}$ of blood was collected in precooled syringes, containing $200 \mu \mathrm{l}$ TrisEDTA (pH 8.65) and $50 \mu \mathrm{l} \mathrm{GSH} \mathrm{(reduced}$ glutathione) $0.05 \mathrm{M}$. Samples were immediately stored in ice, centrifuged at $2500 \times g$ under cooled conditions, and the supernatants frozen at $-80^{\circ} \mathrm{C}$. Adrenaline and noradrenaline concentrations were determined by high 

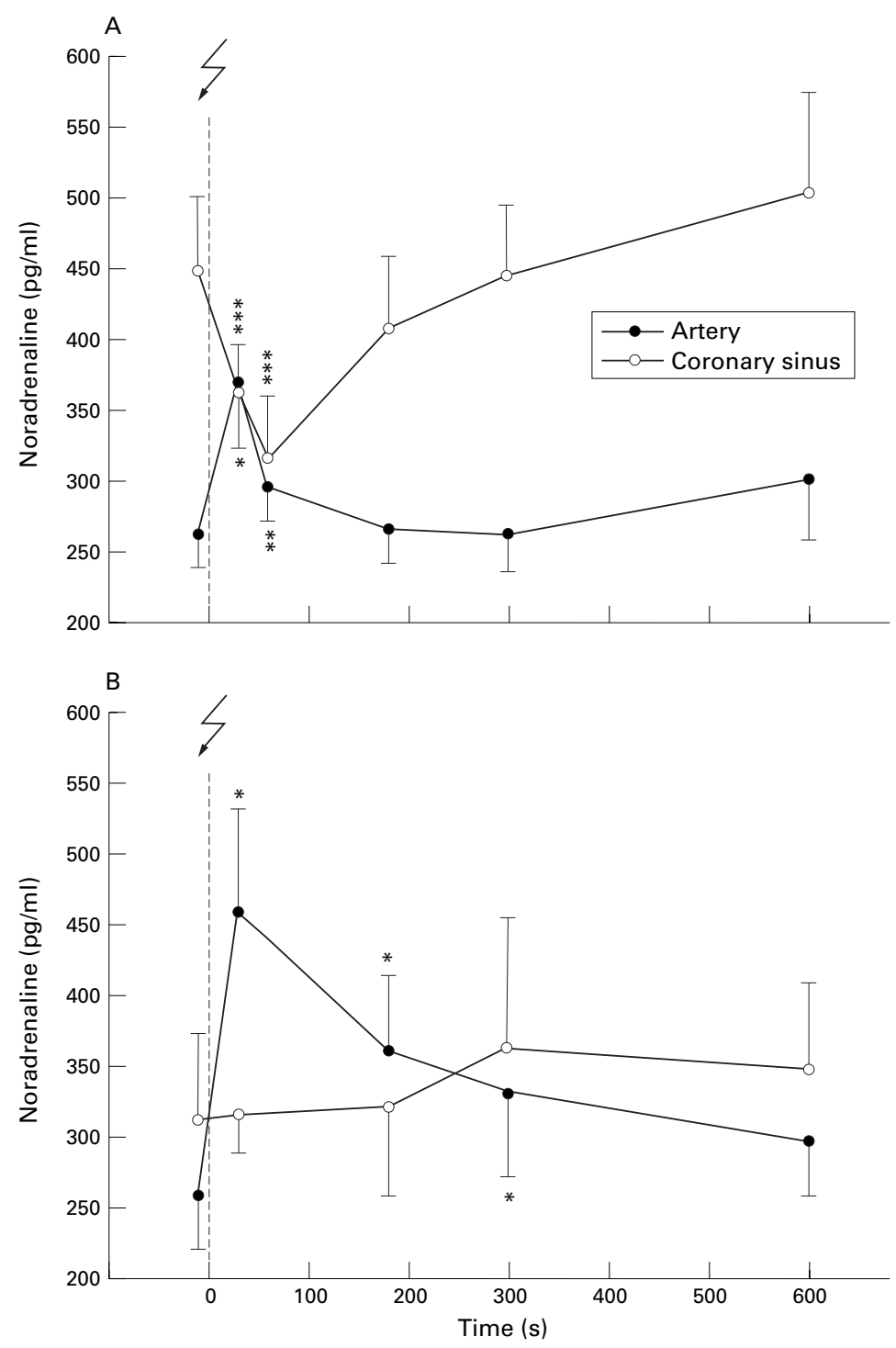

Figure 2 Preshock and postshock noradrenaline concentrations. (A) A marked arterial noradrenaline response to ventricular fibrillation and internal countershock was observed in ICD patients, while coronary sinus noradrenaline decreased. (B) Likewise, external shock delivery for atrial fibrillation increased arterial noradrenaline, but coronary sinus noradrenaline was not significantly affected. Arrows indicate shock application. Values are means, error bars $=$ SEM. ${ }^{\star} p<0.05 ;{ }^{\star \star} p<0.01 ;{ }^{\star \star \star} p<0.001$ v baseline.

performance liquid chromatography with electrochemical detection ${ }^{19}$ (coefficient of variance $<5 \%$, sensitivity $1 \mathrm{pg}$ on column) on a previously described system. ${ }^{20}$ Lactate levels were assessed by lactate oxidase method (Hitachi 717 analyser, Tokyo, Japan; coefficient of variance $0.9 \%$ ).

CALCULATIONS AND STATISTICAL ANALYSIS Data are presented as mean (SD), unless otherwise indicated. Plasma catecholamine and lactate concentrations were compared statistically with corresponding baseline values by the paired $t$ test. Group differences at baseline and for consecutive changes were also examined by $t$ test statistic. Correlation coefficients were calculated by the Pearson method. Probability ( $p$ ) values $<0.05$ defined significance.

According to common practice, transmyocardial substrate gradients were calculated from femoral arterial and coronary sinus plasma concentrations, as a significant release or removal of catecholamines and lactate occurs only in microcirculations of the body, ${ }^{16}$ and circulation time from aortic bulb to femoral artery is short. Percentage myocardial substrate extraction was defined as $100 \times$ (arterial substrate content minus coronary venous substrate content) $\div$ arterial substrate content.

\section{Results}

Shocks were successfully applied in all patients according to the study protocol. Apart from transient rhythm disturbances, no complications were observed. Table 1 shows demographic and clinical features of the two groups.

\section{INDUCED VENTRICULAR FIBRILLATION}

Burst stimulation was employed to induce ventricular fibrillation in 16 patients. $T$ wave shocks $(0.4 \mathrm{~J})$ were applied for induction in 14 patients, 10 of whom required a second attempt. Mean time from initiation of fibrillation to its termination by a single biphasic shock was 15.1 (5.8) seconds. Fibrillation cycle length averaged 182 (38) ms and shock energy 24.7 (10.7) J (range 10 to $34 \mathrm{~J}$ ). In each patient, mean arterial blood pressure fell to 30 $\mathrm{mm} \mathrm{Hg}$ or less during ventricular fibrillation. Early bradycardias (cycle length $\geqslant 1200 \mathrm{~ms}$ ) during the initial five seconds after the shock were observed in 10 patients, ventricular escape rhythms in three, ventricular ectopic beats in 16, and non-sustained ventricular and sinus tachycardia ( $>120$ beats/min) in one. Regularity of sinus rhythm occurred within 3.5 (2.1) seconds after ventricular fibrillation. Sinus rate was 75 (15) beats/min at 30 seconds after ventricular defibrillation, approximating 73 (12) beats/min at baseline (NS).

CARDIOVERSION FROM ATRIAL FIBRILLATION Sinus rhythm was restored in each patient by the first attempted shock with a mean energy of 272 (113) J (range 50 to $360 \mathrm{~J}$ ). Early bradycardias were observed in three patients, and ventricular ectopic beats in five. Regular sinus rhythm was restored within 3.2 (2.6) seconds postshock. Atrial fibrillation with ventricular rates of 80 (21) beats/min was converted to 90 (30) beats/min sinus rhythm.

\section{NEUROHUMORAL AND METABOLIC VALUES AT} BASELINE

At baseline, arterial noradrenaline concentration was $263(128) \mathrm{pg} / \mathrm{ml}$ in the ICD group and $260(113) \mathrm{pg} / \mathrm{ml}$ in patients with atrial fibrillation. Coronary sinus plasma concentration exceeded arterial plasma concentration in both groups. We found no significant differences in noradrenaline concentrations between patients with ventricular arrhythmias and those with atrial fibrillation, but there was a trend towards higher noradrenaline levels in coronary sinus effluent of the former (448 (292) v 382 (279) pg/ml). Intergroup comparison revealed no statistical differences in baseline adrenaline concentrations. Arterial plasma concentration was $44(27) \mathrm{pg} / \mathrm{ml}$ in the ICD group and $52(18) \mathrm{pg} / \mathrm{ml}$ in the atrial fibrillation group. Adrenaline concentrations 

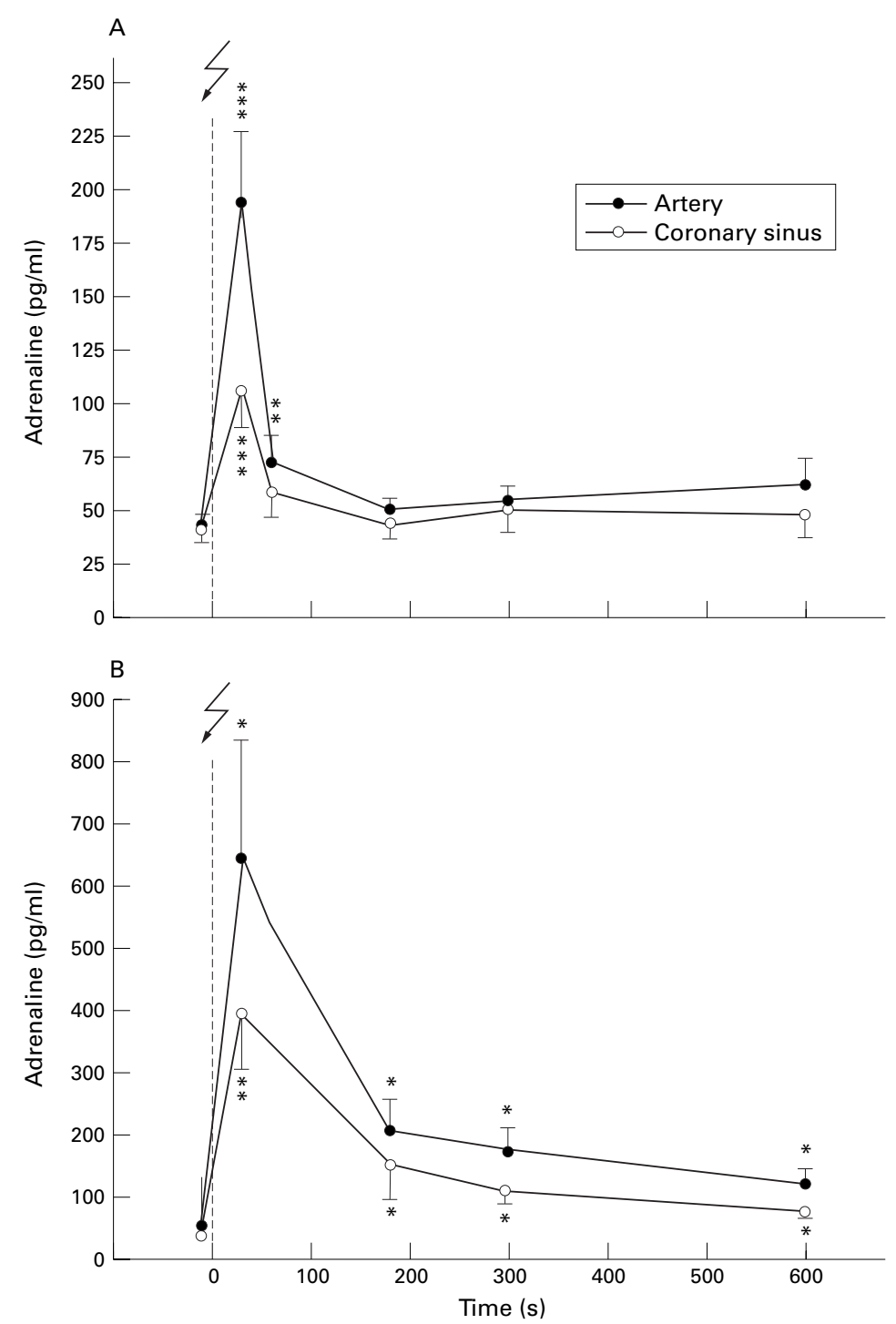

Figure 3 Preshock and postshock adrenaline concentrations. (A) Arterial adrenaline increased in response to internal ventricular defibrillation. (B) After external cardioversion from atrial fibrillation, adrenaline response was even more pronounced. Arterial changes exceeded alterations in coronary sinus levels. Arrows indicate shock application. Values are means, error bars $=$ SEM. ${ }^{\star} p<0.05 ;{ }^{\star *} p<0.01 ;{ }^{\star \star *} p<0.001$ v baseline.

were not significantly higher in arterial than in coronary sinus plasma. No significant correlation was found between catecholamine concentrations and left ventricular ejection fraction, coronary artery disease, or previous myocardial infarction.

All patients showed substantial myocardial extraction of lactate. The lactate extraction ratio was $24 \%$ in the ICD group and $17 \%$ in the atrial fibrillation group (NS). Coronary sinus lactate concentrations were negatively correlated with left ventricular ejection fraction (correlation coefficient $-0.51 ; \mathrm{p}=0.001$ ).

POSTSHOCK METABOLIC CHANGES

Within 30 seconds after ventricular fibrillation and internal shock, the lactate extraction ratio became negative, from $24 \%$ to $-3 \%$ $(\mathrm{p}=0.001)$, reflecting a sharp rise in lactate concentrations in coronary sinus plasma compared with only moderate increases in arterial plasma. The duration of fibrillation correlated with the rise in coronary sinus lactate (correlation coefficient $0.57 ; \mathrm{p}=0.001$ ). The lactate extraction ratio returned to $12 \%$ at one minute $(\mathrm{p}=0.003 v$ baseline) and changes were no longer detectable three minutes after the shock.

In contrast, the lactate extraction ratio was not significantly altered after cardioversion from atrial fibrillation. Transcardiac lactate gradients throughout the study period are shown in fig 1 .

POSTSHOCK NEUROHUMORAL CHANGES

Initiation of ventricular fibrillation and its termination by an internal ICD shock caused increments in arterial concentrations of noradrenaline $(p=0.001)$, whereas coronary sinus values decreased $(\mathrm{p}=0.01)$ immediately after countershock, shifting the positive transcardiac noradrenaline gradient found at baseline towards negative $(p=0.001)$. The greatest reduction in coronary sinus noradrenaline concentrations was reached one minute after the shock. After this, plasma concentrations rapidly approached baseline, and changes were no longer detectable three minutes after the shock (figs 2 and 4). A short lasting, threefold increase in systemic plasma adrenaline occurred following internal shock delivery $(p=0.001)$, exceeding the twofold rise of adrenaline in coronary sinus plasma $(p=0.001)$. As a result, the negative venoarterial plasma concentration gradient increased $(\mathrm{p}=0.001$; figs 3 and 4$)$. The increments in arterial and coronary sinus adrenaline were correlated with the duration of fibrillation (correlation coefficients: $0.52, \mathrm{p}=0.004$, and $0.60, p=0.001$, respectively) and with the defibrillation energy $(0.41, p=0.02$, and 0.40 , $\mathrm{p}=0.03$, respectively).

When ineffective $\mathrm{T}$ wave shocks $(0.4 \mathrm{~J})$ were applied during the ICD test procedure, alterations in plasma catecholamines were not significant.

After external cardioversion from atrial fibrillation, arterial noradrenaline concentrations doubled within 30 seconds of the shock $(p=0.03)$ and remained significantly raised over the fifth minute after the shock. Coronary sinus concentrations were not significantly affected. As a result, the positive transcardiac noradrenaline gradient became negative $(\mathrm{p}=0.01)$ immediately after cardioversion (figs 2 and 4). Initial changes in systemic and coronary sinus noradrenaline did not differ significantly between the groups.

Systemic plasma adrenaline was increased 13-fold by external shock delivery $(p=0.01)$, exceeding the 11 -fold rise in adrenaline concentrations in coronary sinus plasma $(\mathrm{p}=0.004)$ and thereby amplifying the negative venoarterial plasma concentration gradient (figs 3 and 4). The increase in arterial and coronary sinus adrenaline was significantly greater following external shock than after internal application $(p=0.001)$. Despite a rapid decline in plasma concentrations from the peak values, elevations were still observed 10 minutes after cardioversion. No significant correlations were found between neurohumoral changes and left ventricular ejection 



Figure 4 Coronary venous-arterial (c-a) catecholamine differences. (A) Positive transcardiac noradrenaline gradients found at baseline turned negative after termination of ventricular fibrillation by internal shock and after external shock application for atrial fibrillation, indicating a shift from net myocardial release to net uptake. (B) In both clinical settings, negative transcardiac adrenaline gradients were further augmented after the shock procedures, signifying enhanced myocardial adrenaline uptake. Arrows indicate shock application. Values are means, error bars $=$ SEM. ${ }^{\star} p<0.05 ;{ }^{\star \star} p<0.01 ;{ }^{\star \star \star} p<0.001 v$ baseline.

fraction, underlying cardiac disease, analgesic and sedative drug treatment, use of digitalis, or the occurrence of transient rhythm disturbances.

\section{Discussion}

Our study shows considerable systemic sympathetic activation resulting from shock delivery to the human heart, reflected by increments in arterial plasma adrenaline and noradrenaline. These were more pronounced following external than internal shock delivery. In contrast to systemic neurohumoral activation, transmyocardial noradrenaline gradients became negative after both forms of shock application, indicating markedly reduced cardiac sympathetic stimulation.

Noradrenaline serves as a marker of sympathetic nervous activity. Plasma noradrenaline is derived largely from transmitter released by sympathetic nerves, with little contribution from the adrenal medulla. ${ }^{16}$ We observed a rise in systemic plasma noradrenaline after countershock treatment for atrial and ventricular fibrillation, consistent with an increase in sympathetic nerve firing rate.

At baseline, arterial plasma adrenaline was found to be within a typical range for resting conditions, below the threshold for biological effects. ${ }^{21}$ Within 30 seconds of internal and external shock application, adrenaline concentration increased threefold and 11-fold, respectively, and the plasma threshold for cardiovascular and metabolic actions was exceeded. ${ }^{21}$ After a short lasting peak, circulating adrenaline concentrations rapidly approached baseline values in the ICD group, whereas elevations were still detectable after 10 minutes in the atrial fibrillation group. The time courses reflect hormone activity rather than necessarily coinciding with adrenergically mediated actions, which may outlast the decline in the messenger substance. ${ }^{15}$ Under physiological conditions, the bulk of circulating plasma adrenaline has been shown to be derived from adrenal medullary secretion. It is extracted from the plasma by most other organs, producing a negative venoarterial concentration gradient. ${ }^{16}$ Neuronal release of adrenaline into plasma has only been demonstrated in the canine heart, at very high rates of nerve stimulation. ${ }^{22}$ As shown by the enhanced transcardiac adrenaline gradient, the application of dc shocks did not serve as an adequate stimulus for physiologically relevant cardiac adrenaline release in our study.

Simultaneous increases in arterial plasma noradrenaline and adrenaline indicated sympathoneuronal as well as sympathoadrenergic activation. In the ICD group, ventricular fibrillation might have served as a stimulus for increments in sympathetic tone by unloading arterial baroreceptors. ${ }^{12}$ In a previous study a significant change in arterial noradrenaline and adrenaline was found after cardioversion from ventricular tachycardia but not from atrial fibrillation. Sympathetic activation was attributed to hypotension during tachycardia. ${ }^{23}$ Conversely, in another investigation there was no significant increase in plasma catecholamines during ventricular tachycardia before dc shock, but profound changes afterwards. ${ }^{15}$ In our study, the increase in systemic plasma adrenaline after ICD shock showed a modest correlation with the duration of preceding ventricular fibrillation, thereby indicating reflex sympathetic activation due to reduced arterial pressure. However, shock application in atrial fibrillation also produced a marked increase in systemic catecholamine levels, even exceeding the change that occurred after termination of ventricular fibrillation. Thus shock delivery was shown to induce systemic sympathetic activation in the absence of haemodynamic deterioration, independently of baroreflex mechanisms.

There was a moderate correlation between the extent of catecholamine response and the defibrillation energy applied. Support for this is shown by the fact that internal low energy $T$ 
wave shocks did not produce significant changes, while high energy transthoracic shocks led to profound neurohumoral responses. Despite the different current paths, the energy delivered seemed to account for the striking difference in adrenergic activation.

Catecholamine balance varies locally, and as a global index of sympathetic nervous function arterial plasma noradrenaline provides no information on regional nervous sympathetic function. ${ }^{24}$ Our data are the first to show a marked decrease in noradrenaline concentration in coronary sinus effluent after defibrillation from ventricular fibrillation, in contrast to a systemic increase. The cardiac venous noradrenaline concentration reflects the amount released from sympathetic nerve endings plus the amount arriving in the circulating blood, minus reuptake and enzymic metabolism of catecholamines. ${ }^{1625}$ The diffusional flow of noradrenaline is influenced by regional blood flow and the exchange conductivity in the capillary bed. ${ }^{26}$ Owing to these variables, the transmyocardial venoarterial gradient provides a rather rough estimate of cardiac noradrenaline overflow. Nevertheless it has been widely used in clinical settings to characterise changes in regional sympathetic tone, where radiotracer methods are difficult to apply. The positive transmyocardial noradrenaline gradient-indicating cardiac net release of noradrenaline at baseline-became negative after defibrillation from ventricular fibrillation and reflected net uptake. This suggested postshock depression of cardiac sympathetic nervous tone.

Alterations in coronary flow could have influenced coronary sinus noradrenaline concentrations. Reactive hyperaemia is a physiological response during reperfusion after impairment of arterial flow. In a previous investigation, reduced coronary sinus blood flow during ventricular fibrillation was followed by marked hyperaemia 20 seconds after internal defibrillation, with return to prefibrillation values within 30 to 40 seconds. ${ }^{27}$ The data were derived from intraoperative ICD test procedures with fibrillation times of less than 20 seconds and shock energies up to $20 \mathrm{~J}$, comparable with our setting. If correlated with coronary sinus blood flow, cardiac outflow of noradrenaline might be greater during reperfusion than estimated by the coronary venous plasma concentrations. However, in our study the reduction in coronary venous noradrenaline exceeded this hyperaemic period and reached a peak one minute after termination of fibrillation. It was therefore improbable that the observed decrease in coronary venous noradrenaline resulted from altered flow. This assumption is supported by the absence of haemodynamic compromise during cardioversion from atrial fibrillation. Isolated dc shocks have not shown to alter coronary blood flow, ${ }^{28}$ but nevertheless cardioversion caused the positive transcardiac noradrenaline gradient to turn negative.

Impairment of oxidative metabolism might have accounted for the marked decrease in coronary sinus noradrenaline. Net cardiac nor- adrenaline release changes to net uptake as a result of short term myocardial ischaemia. This has been shown in patients with coronary artery disease subjected to atrial pacing stress, and during balloon occlusion of a coronary artery. Myocardial lactate production - a sensitive indicator of anaerobic metabolism ${ }^{29}$ - occurred with concurrent decrements in cardiac noradrenaline release. ${ }^{13} 14$ In our study, a short lasting shift from cardiac lactate extraction to lactate production developed secondary to ventricular fibrillation and internal shock. Increases in coronary sinus lactate concentrations were correlated with arrhythmia duration. Owing to a rapid decline in coronary sinus lactate towards baseline values, net myocardial lactate extraction was re-established as early as one minute after the shock. Transient impairment of myocardial oxidative metabolism was present and served as a possible explanation for decreased cardiac noradrenaline release. In contrast, elective transthoracic cardioversion from atrial fibrillation left myocardial lactate extraction unchanged but induced a similar shift in myocardial noradrenaline balance. This indicated a depressive effect of shocks on cardiac sympathetic activity irrespective of concomitant myocardial ischaemia. The possibility of transient cardiac mitochondrial dysfunction resulting from the shock procedure was also disproved. Animal studies have shown that repetitive shocks reduce aerobic metabolism independently of impaired myocardial oxygen supply. Couples of internal $30 \mathrm{~J}$ shocks delivered to a dog heart, as well as transthoracic $400 \mathrm{~J}$ shocks, resulted in myocardial lactate production that could be detected for several minutes. ${ }^{3031}$ Oxidative metabolism was depressed, in keeping with a primary disturbance of mitochondrial function after discharge..$^{30} \mathrm{In}$ the clinical settings we investigated, a significant depression of oxidative metabolism resulting from the shock delivery was excluded by the absence of myocardial lactate production after cardioversion from atrial fibrillation, and by the time course of lactate changes after ventricular defibrillation.

At present, the underlying mechanism of reduced noradrenaline release from the human heart remains hypothetical. Increased formation of endogenous adenosine has been found during hypoxia and ischaemia, ${ }^{32}$ and may also occur secondary to countershock. ${ }^{33}$ Adenosine inhibits exocytotic release of noradrenaline from sympathetic nerve endings through an $A_{1}$ receptor mediated action, thereby reducing cardiac neurotransmission. ${ }^{34}$ After ventricular fibrillation and countershock application in pigs, postdefibrillatory bradyarrhythmia and haemodynamic depression were prevented by administration of an adenosine antagonist. ${ }^{18}$ Whether endogenous adenosine exerted an antiadrenergic action that accounted for the shock mediated decrease in noradrenaline release in the present study is speculative, especially in the absence of hypoxia and hypoperfusion. This must be the subject of further investigations.

The autonomic nervous system plays an important but complex role in the genesis of cardiac arrhythmias. During ventricular 
fibrillation, sympathetic activation of the myocardium is ascribed to the release of noradrenaline from cardiac sympathetic nerve endings. Shock application was found to decrease cardiac noradrenaline release. After successful defibrillation, this could contribute to postshock depression of cellular function, but might also help to avoid the onset of further fibrillation, as high sympathetic activity is considered to be arrhythmogenic. ${ }^{35}$ Only after the restoration of the coronary circulation does adrenaline secreted by the adrenal gland reach the heart, induce cardiac sympathetic activation and counteract postshock depression after a latent period. If shock application fails to terminate the arrhythmia, reduction in cardiac sympathetic tone could increase the defibrillation threshold during further discharges. ${ }^{10}{ }^{11} \mathrm{In}$ atrial fibrillation the influence of the autonomic nervous system is apparent as vagotonically and sympathotonically evoked paroxysms. ${ }^{36}$ After cardioversion from atrial fibrillation, a depressed neuronal outflow of noradrenaline to the myocardium, paralleled by increases in circulating catecholamines, represents an autonomic imbalance that might influence the stability of sinus rhythm.

LIMITATIONS OF THE STUDY

A limitation of our study is that coronary sinus blood flow was not measured, but this would have hampered the collection of blood samples. Variations in the response to countershock treatment might have occurred in the two clinical settings we investigated. External and internal cardioversion apply different forms of energy pulse and different current routes. Patient populations were different, as were the indications for cardioversion. Thus extrapolation of the results from one setting to the other was made with caution. Analgesia and sedation with midazolam and fentanyl are known to attenuate the overall catecholamine response to stress $^{37-39}$ but cannot explain the differential effects of defibrillation on cardiac and systemic hormones. As the doses applied did not differ significantly between the groups, they cannot account for intergroup variations in sympathetic response. The use of etomidate does not appear to influence plasma catecholamines. ${ }^{37}$ Several patients were on chronic digitalis treatment, which has been shown to reduce the sympathetic overactivity in heart failure and to normalise impaired baroreflex mediated mechanisms. ${ }^{40}$ Amelioration of autonomic dysfunction by digitalis in some patients with moderate heart failure could have reduced their baseline catecholamine release without an adverse effect on shock evoked responses.

\section{CONCLUSIONS}

Shock application induces systemic sympathetic activation as reflected by enhanced adrenomedullary and sympathetic neural outflow of neurotransmitters to the circulation. In contrast, cardiac sympathetic activity is depressed. This depression is not mediated by hypoxaemia or ischaemia, but results from electrical discharge to the myocardium.

1 Eysmann SB, Marchlinski FE, Buxton AE, et al. Electrocardiographic changes after cardioversion of ventricular arrhythmias. Circulation 1986;73:73-81.

2 Waldecker B, Brugada P, Zehender M, et al. Dysrhythmias fter direct-current cardioversion. Am f Cardiol 1986; 57:120-3.

3 Yabe S, Smith WM, Daubert JP, et al. Conduction disturbances caused by high current density electric fields. Circ Res 1990;66:1190-203.

4 Anderson GJ, Reiser J. Electrophysiologic changes induced by external transthoracic countershock. $\mathcal{f}$ Electrocardiol by external tran

5 Jones JL, Lepeschkin E, Jones RE, et al. Response of cultured myocardial cells to countershock-type electric field stimulation. Am f Physiol 1987;235:H214-22.

6 Pansegrau DG, Abboud FM. Hemodynamic effects of ventricular defibrillation. $\mathcal{F}$ Clin Invest 1970;49:282-97.

7 Ten Eick RE, Wyte SR, Ross SM, et al. Post-countershock arrhythmias in untreated and digitalized dogs. Circ Res 1967;21:375-90.

8 Cobb FR, Wallace AG, Wagner GS. Cardiac inotropic and coronary vascular responses to countershock. Evidence for excitation of intracardiac nerves. Circ Res 1968;23:731-42.

9 Yu JCL Luer MR, Young $C$ et al. Ventricular pacing Y J , Lauer MR, Young C, et al. Ventricular pacing threshold and refractoriness after defibrillation shocks in Heart 7 1996;131:1121-6.

10 Ruffy R, Schechtman K, Monje E. Adrenergically mediated variations in the energy required to defibrillate the heart: observations in closed-chest, nonanaesthetized dogs. Circulation 1986;73:374-80.

11 Rattes MF, Sharma AD, Klein GJ, et al. Adrenergic effects on internal defibrillation threshold. Am $\mathcal{F}$ Physiol 1987;253: H500-6.

12 Reison D, Oliver JA, Sciacca RR, et al. Release of norepinephrine from sympathetic nerve efferents by bilateral carotid occlusion. Am F Physiol 1983;245:H635-9.

13 Remme WJ, Kruyssen DA, Look MP, et al. Systemic and cardiac neuroendocrine activation and severity of myocardial ischemia in humans. F Am Coll Cardiol 1994;23:82-91.

14 Richardt G, Muench G, Neumann FJ, et al. Systemic and cardiac catecholamines during elective PTCA and during immediate PTCA for acute myocardial infarction. Basic Res Cardiol 1997;92:52-60.

15 Morady F, DiCarlo LA, Halter JB, et al. The plasma catecholamine response to ventricular tachycardia induction and external countershock during electrophysiologic tion and external countershock during el.

16 Esler M, Jennings G, Lambert G, et al. Overflow of catecholamine neurotransmitters to the circulation: source, fate and functions. Physiol Rev 1990;70:963-85.

17 Esler M, Jennings G, Korner P, et al. Measurement of total and organ-specific norepinephrine kinetics in humans. $\mathrm{Am}$ f Physiol 1984;247:E21-2.

18 Wesley RC, Porzio D, Sadeghi M. Effect of selective A1 adenosine receptor antagonism of postdefibrillation cardio-
vascular depression: evidence for an antiadrenergic role of vascular depression: evidence for an antiadrenergic role
endogenous adenosine. Cardiovasc Res 1993;27:129-33.

19 Hjemdahl P. Catecholamine measurements in plasma by high-performance liquid chromatography with electrochemical detection. Methods Enzymol 1987;142:521-34.

20 Raasch W, Grobecker H, Kees F. Influence of ciprofloxacin on brain metabolism of biogenic amines in the rat. Pharm Pharmacol Lett 1992;2:153-6.

21 Clutter WE, Bier DM, Shah SD, et al. Epinephrine plasma metabolic clearance rates and physiological thresholds for metabolic and hemodynamic actions in man. $\mathcal{F}$ Clin Invest 1980;66:94-101.

22 Peronnet F, Nadeau R, Boudreau G, et al. Epinephrine release from the heart during left stellate ganglion stimulation in dogs. Am f Physiol 1988;254:R659-62.

23 Salerno DM, Katz A, Dunbar DN, et al. Serum electrolytes and catecholamines after cardioversion from ventricular tachycardia and atrial fibrillation. PACE 1993;16:1862-71.

24 Esler M. Clinical application of norepinephrine spillover methodology: delineation of regional human sympathetic nervous responses. Pharmacol Toxicol 1993;73:243-53.

25 Manoach M, Varon D, Erez M. The role of catecholamines on intercellular coupling, myocardial cell synchronization on intercellular coupling, myocardial cell synchronization 1995;147: 181-5.

26 Cousineau D, Goresky CA, Bach GG, et al. Effect of $\beta$-blockade on in vivo norepinephrine release in canine heart. Am f Physiol 1984;246:H283-92.

27 Runsiö M, Rosenqvist M, Bergfeldt L, et al. Cardiac outflow of endothelin, neuropeptide $\mathrm{Y}$ and noradrenaline in relation to hyperemia in coronary sinus flow following electrical conversion of induced ventricular fibrillation in man. Eur Heart $\mathcal{F}$ 1995;16:1925-9.

28 Lucy SD, Jones DL, Klein GJ. Effects of defibrillation shocks delivered over a major coronary artery. PACE 1992; 15:1711-19.

29 Gertz EW, Wisneski JA, Neese R, et al. Myocardial lactate extraction: multi-determined metabolic function. Circulation 1980;61:256-61.

30 Gaba DM, Maxwell MS, Merlone S, et al. Internal countershock produces myocardial damage and lactate production without myocardial ischemia in anesthetized dogs. Anesthesiology 1987;66:477-82. 
31 Trouton TG, Allen JD, Adgey AAJ. Oxidative metabolism and myocardial blood flow changes after transthoracic DC

countershocks in dogs. Eur Heart f 1992;13:1431-40.
32 Haneda T, Ichihara K, Abiko Y, et al. Release of adenosine and lactate from human hearts during atrial pacing in patients with ischemic heart disease. Clin Cardiol 1989;12: $76-82$

33 Ito M, Pride HP, Zipes DP. Defibrillating shocks delivered to the heart impair efferent sympathetic responsiveness. Circulation 1993;88:2661-73.

34 Richardt G, Waas W, Kranzhöfer R, et al. Adenosine inhibits exocytotic release of endogenous noradrenaline in rat
heart: a protective mechanism in early myocardial ischemia. Circ Res 1987;61:117-23.

35 Lown B, Verrier RL. Neural activity and ventricular fibrillation. N Engl F Med 1976;294:1165-70.
36 Coumel P. Paroxysmal atrial fibrillation: a disorder of autonomic tone? Eur Heart f 1994;15(suppl A):9-16.

37 Crozier TA, Beck D, Schlaeger M, et al. Endocrinological changes following etomidate, midazolam, or methohexital for minor surgery. Anaesthesiology 1987;66:628-35.

38 Glisson SN. Investigation of midazolam's influence on physiological and hormonal responses to hypotension. $\mathcal{F}$ Cardiovasc Pharmacol 1987;9:45-50.

39 Chraemmer-Jorgensen B, Hertel S, Strom J, et al. Catecholamine response to laryngoscopy and intubation. Anaesthesia 1992;47:750-6.

40 Krum H, Bigger T, Goldsmith RL, et al. Effect of long-term digoxin therapy on autonomic function in patients with chronic heart failure. F Am Coll Cardiol 1995;25:28994 